\title{
A Model for Predicting the Amount of Urine in the Bladder Based on App-generated Tracking Data
}

\author{
Pascal Fechner ${ }^{*}$, Jannik Lockl ${ }^{\dagger}$, Nicolas Ruhland*, Tristan Zürl*, and Till Zwede ${ }^{\dagger}$ \\ ${ }^{*}$ Faculty of Engineering ${ }^{\dagger}$ Faculty of Law, Business \& Economics \\ University of Bayreuth \\ Bayreuth, Germany \\ Email: \{pascal.fechner, lockl.jannik, nicolas.w.ruhland, tristan.zuerl, till.zwede\}@ uni-bayreuth.de
}

\begin{abstract}
Incontinence patients suffering from neurogenic bladder dysfunction lack information about the filling level of their urinary bladder. A real-time prediction of the filling level of their bladder could support them managing their daily routines. In this study, we developed a system that predicts the bladder filling level based on user-tracked fluid intake. The system collects and analyzes the data to predict the current filling level of the bladder. Displayed in an app, users can optimize their micturition frequency receiving an alert when a critical level is reached. In the same way users can compare the predicted- and their target filling level.
\end{abstract}

Index Terms-app, chronic disease management, data analytics, eHealth, inContAlert, incontinence, prediction

\section{INTRODUCTION AND MOTIVATION}

Neurogenic bladder dysfunction is a disease that affects, for example, people suffering from Multiple Sclerosis or Paraplegia. Respective patients do not feel the filling of their bladder anymore. They must empty their bladder according to manual drinking or time protocols and a good guess, how full their bladder is [5]. The body, however, does not process the fluids consumed in a way that patients could always empty their bladder the same time-span after an intake and expect the same amount to be urinated. The processing of fluids is affected by drinking and eating intakes, as well as fluid loss through, for example, breathing, sweating, dejection, and micturition. Depending on different parameters such as time of the day, physical state, and hydration status, the speed and amount of fluid processed through the body differs [2].

If patients do not empty their bladder in time, they run the risk of an uncontrolled loss of urine. Additionally and even worse, an overfilled bladder can cause a backflow of the urine and consequent damage of the kidneys. Reducing these risks, patients tend to empty their bladder in a high frequency (i.e., after three hours) regardless of whether the bladder is full or not. The bladder, in contrast, needs to be filled up regularly to not degenerate. Summarized, the missing knowledge of patients on how full their bladder is leads to the risk of uncontrolled loss of urine, damage to the kidneys, and

978-1-7281-6215-7/20/\$31.00 @2020 European Union a degenerating bladder. All of this leads to stigmatization of the patients [9], is health threatening, and reduces a patient's quality of life [4] [7].

\section{EXPERIMENTAL SETUP}

Digital technology and advanced analytics can help to derive the real-time filling level of the bladder. Such health-tech could provide the information needed for improving micturition management. The study aims at predicting the filling level of the urinary bladder of a person as accurate as possible by recording hydration-relevant processes of the body. To develop such a system, we derived requirements for the software application and the data used for the measurement [3]. Data must be collected as continuously and thoroughly as possible. Data generation must be integrated into the daily routines of the test persons in a simple way to prevent fragmentary data [8]. Other options are to track the intake and loss through an observing person, or to fully-automate the tracking on the base of sensors monitoring intake and loss to reduce the risk of logging mistakes. Staffing an observing person would have compromised the convenience of our experiment. Deploying an extensive sensor-based monitoring system, again, was not feasible as such setting cannot be used in the real-world. For this reason, we decided to develop a smartphone app as a medium for convenient recording of fluid intake, such as eating and drinking, and fluid loss, such as micturition or heavy physical activity [2]. Fig. 1 shows the user interface of above mentioned app with three different logging options.

Qualitative and quantitative options exist to track fluid intake and loss. To minimize random effects within our experimental setup, we determined the conditions for the manual data collection precisely. Fluid intake and micturition were recorded with measuring jugs that had a tolerance range of $10 \mathrm{ml}$ each. To assure comparable conditions, every proband logged the amount of fluid intake at the moment the intake happened. The weight loss of sweating due to physical activity was determined by a before-and-after comparison. The experiments were limited to males without bladder dysfunction between 20 and 30 years of age. Besides, we supposed that every proband emptied the bladder fully so that no fluid re- 


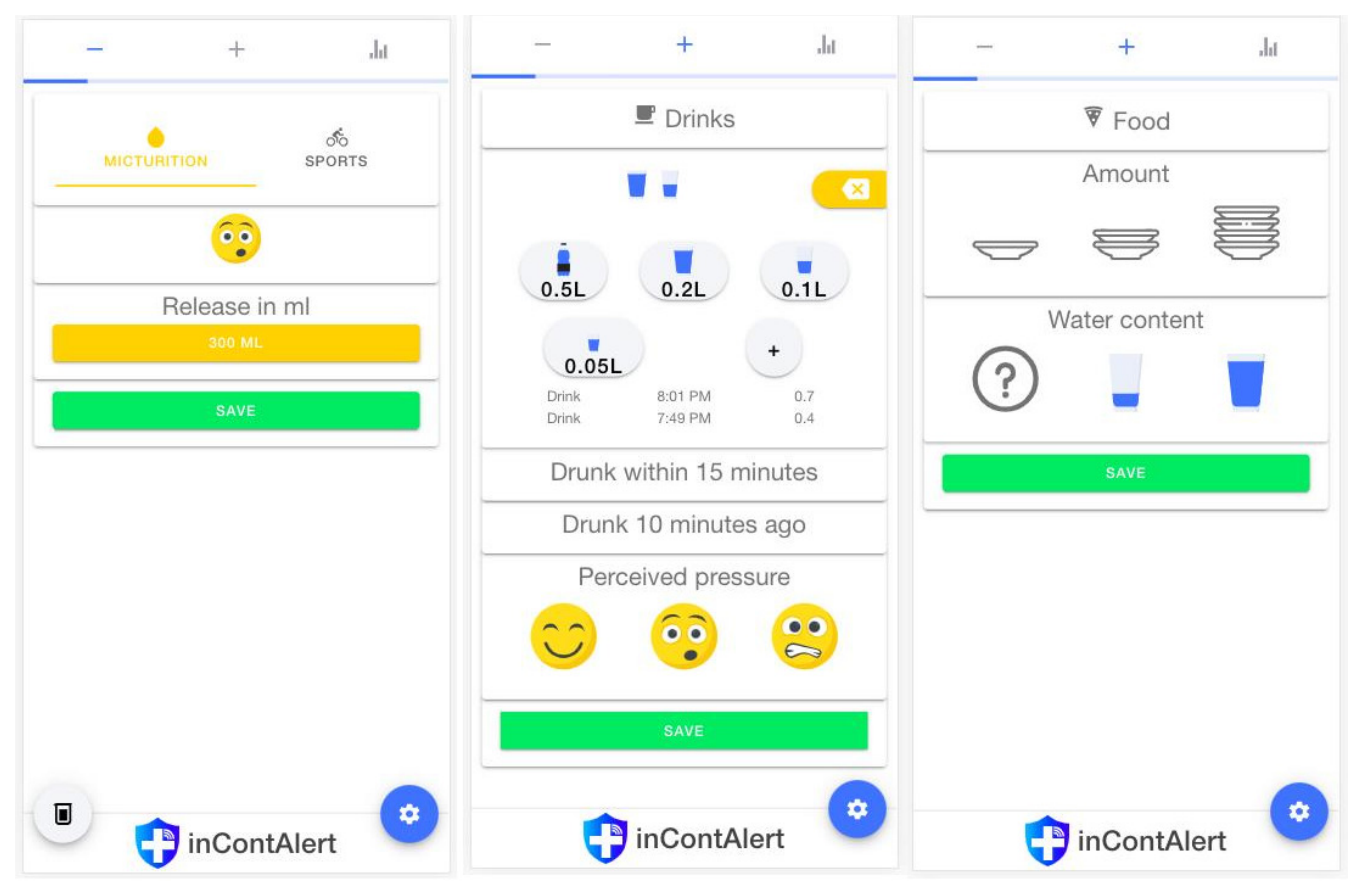

Figure 1: Graphical User Interface with the Functionalities Micturition Tracking, Fluid Intake, Food Intake

mained in the bladder. All probands were constrained to drink exclusively non-alcoholic beverages and respect a normal diet. Each participant logged data during the same seven complete days. The daily routines of the persons did not include any strenuous physical work, and they were in the same city to ensure a comparable environment. As the experimental days were not subsequent, the logging began just after the first micturition of a day to have a common starting point.

\section{DATA ANALYSis}

\section{A. Mathematical Foundation}

To derive the filling level of the urinary bladder, we mathematically described the filling by the Gaussian curve [1] $\Theta(x)$ (c.f., Formula (1)), where $\mu$ is set to be the mean delay and $\sigma$ to be the standard deviation. We optimized the variables $\mu$ and $\sigma$ for the first test person and applied them to the others.

$$
\Theta(x)=\frac{1}{\sigma \sqrt{2 \pi}} e^{-\frac{1}{2}\left(\frac{x-\mu}{\sigma}\right)^{2}}
$$

\section{B. Implementation}

The kidneys start to process the fluid intake to urine after a certain time delay. During this delay no fluid is processed in the mathematical model. After $\mu-3^{*} \sigma$ minutes the processing by the Gaussian curve starts and urine enters the bladder. In our experimental setting we found that $\mu$ was in the range of $[40 ; 60]$ minutes and $\sigma$ in the range of $[3 ; 6]$ minutes for all probands. Every minute the integral over the curve is evaluated, until the overall integral of the Gaussian is equal to the amount of fluid taken in. For every proband a fixed amount of fluid loss for each minute was deducted. This value was determined experimentally to be per minute in the range of $[0 ; 0.4] \mathrm{ml}$ for all probands.

Every fluid intake causes a Gaussian curve based on the Formula (1) to be processed with the above mentioned delay. Similarly the food intake induces a Gaussian curve $\Theta(x)$ to be processed with a time delay but it was found to be irrelevant for the prediction accuracy. Further, food intake should influence the fluid absorption by a certain delay, but we found that this does not influence the prediction significantly. The sport data accounted for an additional deduct on top of the natural fluid loss per minute. Depending on the intensity, we experimentally determined an additional loss between the range of $[5 ; 30] \mathrm{ml}$ per minute for low intensity, $[5 ; 30] \mathrm{ml}$ per minute for medium intensity, and [5;30] $\mathrm{ml}$ per minute for high intensity. In the case of dehydration as a result of sport or natural loss, the negative balance had to be first balanced out by fluid intake before the algorithm started again to process the fluid intake to urine.

Every time a micturition takes place, the bladder filling level is reset to $0 \mathrm{ml}$. The filling level still increases after a micturition in the case that remaining fluid is still being processed [1]. To account for natural filling restrictions through kidney capacities, we limited the maximum filling rate of the bladder to the range of $[4 ; 8] \mathrm{ml}$ per min. Each day, we analyzed the environment and adapted the parameters influenced by the environment, such as the loss of fluid that is influenced by the environmental temperature. We adapted the parameters manually as an automated adaptation of influencing variables was out of scope for our study. Fig. 2 depicts the exemplary filling curve of the bladder of a proband during the course of a day. The bladder fills according to the Gaussian Curve $\Theta$ and 


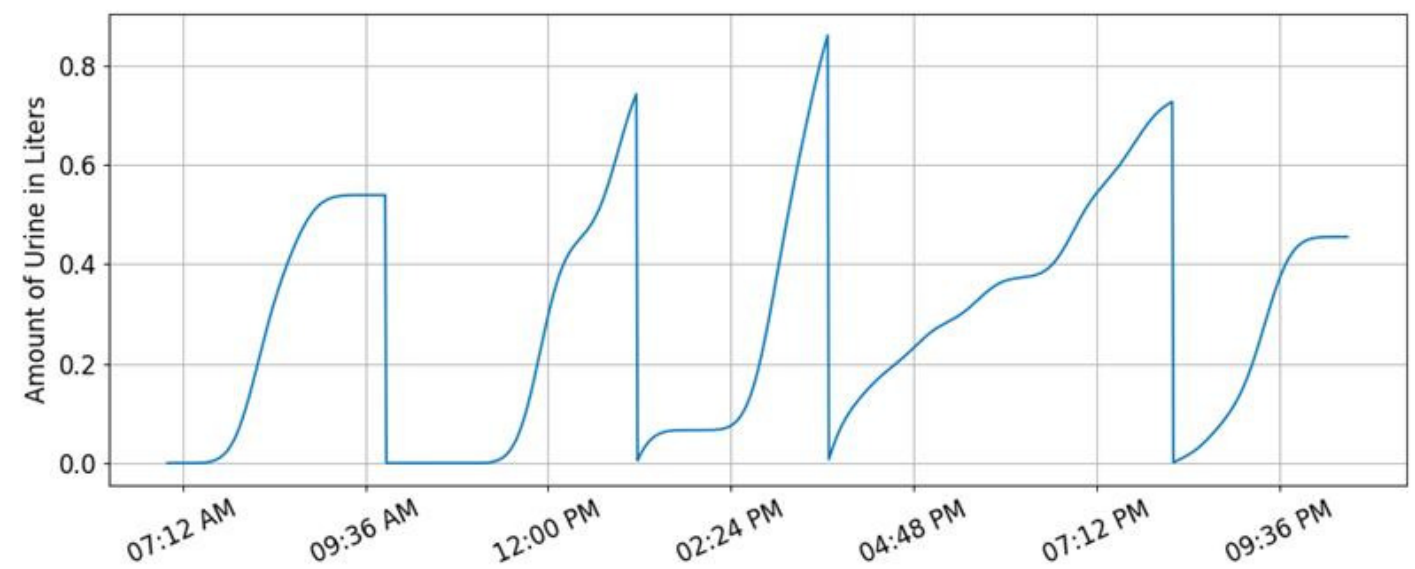

Figure 2: Predictions of the Filling Curves of the Bladder a Proband During the Course of a Day

our predetermined parameters, and empties when a micturition takes place. Multiple Gaussian curves can overlap and add up onto each other accounting for the deformed lines in Fig. 2.

\section{Evaluation}

To evaluate the model, our algorithm compares the predicted filling level with the amount of urine collected by the probands in the measuring jug. Using four days (i.e., test days) of the first proband, we determined experimentally which parameters resembled the reality best. To validate our assumptions, we applied the so-determined parameters to the remaining days (i.e., validation days) of the first and all other probands. We then calculated the relative error $\eta$ of the validation data set. Hereby, $\eta$ is calculated using Formula (2) where $\delta_{\text {true }}$ is the value measured by the probands and $\delta_{\text {pred }}$ is the prediction made by the algorithm:

$$
\eta=\frac{\delta_{\text {true }}-\delta_{\text {pred }}}{\delta_{\text {true }}}
$$

$\eta$ of a validation day of one proband was calculated using the mean of each $\eta$ of all micturitions during that day. Furthermore, $\eta$ of all validation days of one person was calculated using the mean of all the $\eta$ of all validation days for this proband. After adjusting parameters to be optimal within their ranges for a respective day, $\eta$ of the test days was .29. After applying these specific parameters to the validation days, an $\eta$ of .33 was found for the proband whose data was partially used for the test days. When applied to all the other probands, $\eta$ was .49.

The test day with the best prediction for one proband reached an $\eta$ of .0709 . Optimizing the validation days with the ranges found in the test days resulted in a mean $\eta$ of 0.38 for all test persons.

\section{CONCLUSiON}

We developed a system, which predicts the bladder filling level based on user-tracked fluid intake and loss. The system collects and analyzes the data to predict the current filling level of the bladder. We showed that with an individual calibration acceptable relative prediction errors of less than $10 \%$ are possible. Displayed in an app, users can optimize their micturition frequency comparing the predicted and their target filling level. For patients who must rely on such a system to manage their daily routines, the predictive system can add great value. Variables of disturbance seem to be the environment leading to altered loss of fluids and the probanddependent tracking due to possible logging mistakes. Future research is invited to validate our findings with additional data, and to develop systems of fully-automated tracking of fluid intake and loss. Furthermore, examining the environmental factors influencing the processing of fluids in the body is a promising path to further explain deviations and finally improve the prediction.

\section{REFERENCES}

[1] Kurihara, Y., Yamasaki, T., Kaburagi, T., Kumagai, S., and Matsumoto, T., Model of Urine Accumulation in the Bladder and Method for Predicting Unconstrained Urine Volume Based on Absorption Spectrum of Urine, Volume 8 IEEE Access, Pages 69368 - 69377, 2020.

[2] Popkin, B.M., D’Anci, K.E., Rosenberg, I.H., Water, hydration and health, Volume 68, Nutrition Reviews, Pages 439-458, 2010.

[3] Liu, D., Hsieh, H.-H., Tsay, W.-D., Ke, M.-H., Analysis on Functional Demand of Mobile-Health APP for Elders, 3rd ed. IEEE 2nd Eurasia Conference on Biomedical Engineering, Healthcare and Sustainability, 2020.

[4] Niestoruk, L., Beuth, T., Petry, K., Balzer, M., Stork, W., Mueller-Glaser, $\mathrm{K}$., A concept for wearable long-term urinary bladder monitoring with ultrasound. Feasibility study, 3rd ed. IEEE, 5th European DSP Education and Research Conference, 2012.

[5] DeMaagd, G.A., Davenport, T.C., Management of Urinary Incontinence., Pharmacy and Therapeutics 37, 345-361, 2012.

[6] Irwin, D.E., Kopp, Z.S., Agatep, B., Milsom, I., Abrams, P., Worldwide prevalence estimates of lower urinary tract symptoms, overactive bladder, urinary incontinence and bladder outlet obstruction, BJU International 108(7), 1132-1138, 2011.

[7] Rizvi R.M., Ather, M.H., Assessment of Urinary Incontinence in Adult Patients, IntechOpen, Synopsis in the Management of Urinary Incontinence, 2017.

[8] Dou, K., Yu, P., Deng, N., Liu, F., Guan, Y.P., Li, Z., Ji, Y., Du, N., Lu, X., Duan, H., Patients' Acceptance of Smartphone Health Technology for Chronic Disease Management: A Theoretical Model and Empirical Test, JMIR mHealth and uHealth 5(12), 2017.

[9] Toye, F., Barker, K.L., A meta-ethnography to understand the experience of living with urinary incontinence: 'is it just part and parcel of life?', BMC Urology 20(1), 2020. 